\title{
Quantum correlations from local amplitudes and the resolution of the Einstein-Podolsky-Rosen nonlocality puzzle
}

\author{
C. S. Unnikrishnan* \\ Gravitation Group, Tata Institute of Fundamental Research, \\ Homi Bhabha Road, Mumbai - 400 005, INDIA, \\ and \\ NAPP Group, Indian Institute of Astrophysics, \\ Koramangala, Bangalore - 560 034, INDIA \\ Prepared for presentation at the International Conference on \\ Quantum Optics, ICQO'2000, Minsk, Belarus
}

\begin{abstract}
The Einstein-Podolsky-Rosen nonlocality puzzle has been recognized as one of the most important unresolved issues in the foundational aspects of quantum mechanics. We show that the problem is more or less entirely resolved if the quantum correlations are calculated directly from local quantities which preserve the phase information in the quantum system. We assume strict locality for the probability amplitudes instead of local realism for the outcomes, and calculate an amplitude correlation function. Then the experimentally observed correlation of outcomes is calculated from the square of the amplitude correlation function. Locality of amplitudes implies that measurement on one particle does not collapse the companion particle to a definite state. Apart from resolving the EPR puzzle, this approach shows that the physical interpretation of apparently 'nonlocal' effects like quantum teleportation and entanglement swapping are different from what is usually assumed. Bell type measurements do not change distant states. Yet the correlations are correctly reproduced, when measured, if complex probability amplitudes are treated as the basic local quantities. As examples we derive the quantum correlations of twoparticle maximally entangled states and the three-particle GHZ entangled state.
\end{abstract}

${ }^{*}$ Email address: unni@tifr.res.in 


\section{Introduction}

Quantum nonlocality as manifested in the EPR correlations has been, without doubt, the most important unresolved problem in the foundational aspects of physics. All experiments on quantum correlations and test of Bell's inequality, and their present interpretations based on the multiparticle wavefunction in quantum mechanics suggest that there is nonlocality. Yet, we understand neither the nature of this nonlocality nor the physical mechanism that establishes the nonlocal correlations. The situation is somewhat akin to that in the earlier part of the last century when the ether was thought to be a necessary concept for the propagation of electromagnetic waves, yet something not apparent or detectable.

Sixty five years ago, Einstein, Podolsky and Rosen (EPR) [1] addressed the question whether the wave-function represented a complete description of reality in quantum mechanics, and argued that it didn't. The crucial and essential assumption in their argument was strict nonlocality, in the spirit of special relativity. Also, they had considered and included the concept of objective reality in the analysis. If the value of an observable was predictable with certainty without a measurement, the observable had a physical reality according to EPR. Their assertions lead to attempts at constructing a hidden variable theory that was more complete. Bell's analysis of the EPR problem in the early sixties established the Bell's inequalities obeyed by any local hidden variable theory for the correlations of entangled particles [2]. Quantum mechanical correlations calculated using the entangled wave-function and spin operators violate these inequalities. Various experiments have established beyond doubt that there cannot be a viable local realistic hidden variable description of quantum mechanics [3]. Further, these results also have been interpreted as evidence for nonlocal influences in quantum measurements involving entangled particles. Since no instruction set carried by the particles from their source of origin (possibly with the addition of several local hidden variables) can manage to create the correct correlations observed in experiments, the only way out seems to be that measurement of an observable on one of the particles in an entangled pair seems to convey the result of this measurement instantaneously to the other particle resulting in the correct behaviour of the other particle during a measurement on the second particle. In the quantum mechanical terminology, the measurement of an observable on one of the particles collapses the entire wave-function instantaneously and nonlocally and the second particle acquires a definite value for the same observable, consistent with the relevant conservation law. The no signalling theorems in this context prohibit any faster than light signalling using this feature, and therefore signal locality is not violated. But the stronger requirement of Einstein locality is violated. We seem to be stuck with the puzzling nonlocality which is probably the deepest mystery in the behaviour of entangled systems.

Accepting the concept of nonlocality without being able to understand its nature is already a disturbing feature. There is also serious conflict with the spirit of relativity. 
If one measurement precede the other in one frame, one can always find a moving frame in which the converse it true, the second measurement preceding the first [1]. Therefore, one cannot attribute cause and effect relationships for measurements causing nonlocal collapse.

It turns out that the long-standing problem of EPR nonlocality is resolved by a simple quantum step that is physically well motivated [5]. The crucial new idea is to incorporate the fact that quantum systems have 'wave aspects' in their behaviour and all calculations should take into account the phase relationships (coherence) that might be there in the multiparticle system. If locality is assumed at the level of probability amplitudes, and if the correlation is calculated directly from these amplitudes the correct quantum correlations emerge. This means that the EPR definition of objective reality was too restrictive. There is objective reality, but that is at the level of quantum phases and not at the level of eigenvalues. The quantum correlation is encoded in the relative phase appropriate for the problem. In the local hidden variable theories the correlations are calculated from eigenvalues and this procedure does not preserve the phase information. The situation has some analogy to the description of interference in quantum mechanics. Any attempt to reproduce the interference pattern using locality and the information on 'which-path' will fail since the phase information is lost or modified in such an attempt.

\section{The solution of the EPR nonlocality puzzle}

Consider the breaking up of a correlated state as in the standard Bohm version of the EPR problem [6]. The two-particle state is described by the wave function

$$
\Psi_{S}=\frac{1}{\sqrt{2}}\{|1,-1\rangle-|-1,1\rangle\}
$$

where the state $|1,-1\rangle$ is short form for $|1\rangle_{1}|-1\rangle_{2}$, and represents an eigenvalue of +1 for the first particle and -1 for the second particle if measured in any particular direction. $\Psi_{S}$ is inherently nonlocal, describing both particles together, even when they are far apart in space-like separated regions.

Two observers make measurements on these particles individually at space like separated regions with time stamps such that these results can be correlated later through a classical channel. We assume that strict locality is valid at the level of probability amplitudes [5]. A measurement changes probability amplitudes only locally. Measurements performed in one region do not change the magnitude or phase of the complex amplitude for the companion particle in a space-like separated region. The local setting of the polarizers, analyzers, Stern-Gerlach analyzers etc. (collectively denoted as analyzer) is represented by $\mathbf{a}$ and $\mathbf{b}$ for the two distant apparatus. These could be the directions of the analyzers, for example. Since we need to deal with correlated particles which may have a definite phase relationship at source (when 
the particles are produced together, for example) we introduce internal variables associated with each particle. We denote these variable as $\phi_{1}$ and $\phi_{2}$. Their values are unaltered once the particles are separated. Measurement on one particle does not change the value of this internal variable for the other particle. The assumption of locality is that the amplitudes (as opposed to eigenvalues) are functions of only these local variables.

We now state the assumptions (1 and 2$)$ and some related comments $(3,4$,and 5$)$ :

1. The local amplitude for the first particle $C_{1}$ that decide the passage of the particle through an analyzer depends only on the local variables a and $\phi_{1}$. Similarly $C_{2}$ depends only on $b$ and $\phi_{2}$. If we denote the passage as + and the alternate outcome as -, then the statement of locality is for the relevant amplitudes is

$$
C_{1 \pm}=C_{1 \pm}\left(\mathbf{a}, \phi_{1}\right), \quad C_{2 \pm}=C_{2 \pm}\left(\mathbf{b}, \phi_{2}\right)
$$

2. The correlations of the particles are encoded in the difference of the internal variables $\phi_{1}$ and $\phi_{2}$. If the particles have perfect correlations at source then all the pairs in the ensemble have the same value for the difference $\left|\phi_{1}-\phi_{2}\right|=\phi_{0}$.

3. We do not make any assumption on determinism. Given the initial values of the internal variables $\phi_{1}$ and $\phi_{2}$, we do not attempt to make any prediction of the eigenvalues that would be measured in each run of the experiment.

4. We do not assume any hidden variable in the problem. The variables $\phi_{1}$ and $\phi_{2}$ are associated with the particles and they could be considered as hidden variables in a formal sense, though these values are not measurable since only relative phases are measurable.

5. We will also state the locality at the level of the eigenvalues, though we do not use this in the calculation. For observables $A$ and $B$,

$$
A\left(\mathbf{a}, \phi_{1}\right)= \pm 1, \quad B\left(\mathbf{b}, \phi_{2}\right)= \pm 1
$$

This is the same locality assumption as in local realistic theories [2]. But, this has a meaning different from its meaning in standard local realistic theories. Here, this means that the outcomes, when measured, depend only on the local setting and the local internal variable. There is no objective reality to $A$ and $B$ before a measurement. There is objective reality to $\phi_{1}$ and $\phi_{2}$, but there is no way to observe these absolute phases.

Note that $\phi$ is not a dynamical phase evolving as the particle propagates. It is an internal variable whose difference (possibly zero) remains constant for the particles of the correlated pair. The value of $\phi$ can vary from particle to particle, but the relative 
phase $\phi_{0}$ between the two particles in all correlated pairs is constant. Consider $\phi$ as a reference for the particles to determine the angle of a polarizer or analyzer encountered on their way, locally.

The first particle encounters analyzer 1 kept at an angle $\theta_{1}$ with respect to some global direction. We denote this angle of the analyzer with reference to $\phi$ as $\theta$. Similarly, the second particle which has the internal phase angle $\phi+\phi_{0}$, where $\phi_{0}$ is a constant, encounters the second analyzer oriented at angle $\theta_{2}$ at another space-like separated point. Let the orientation of this analyzer with respect to the internal phase angle of the second particle is $\theta^{\prime}$. We have $\theta-\theta^{\prime}=\theta_{1}-\theta_{2}+\phi_{0}$.

An experiment in which each particle is analyzed by orienting the analyzers at various angles $\theta_{1}$ and $\theta_{2}$ is considered next. At each location the result is two-valued denoted by $(+1)$ for transmission and $(-1)$ for absorption of each particle, for any angle of orientation. The classical correlation function, which is also the experimenter's correlation function, $P(\mathbf{a}, \mathbf{b})=\frac{1}{N} \sum\left(A_{i} B_{i}\right)$ satisfies $-1 \leq P(\mathbf{a}, \mathbf{b}) \leq 1$. Here $(\mathbf{a}, \mathbf{b})$ denotes the two directions along which the analyzers are oriented and $A_{i}$ and $B_{i}$ are the two valued results. We note that $P(\mathbf{a}, \mathbf{b})$ denotes the average of the quantity (number of detections in coincidence - number of detections in anticoincidence), where 'coincidence' denotes both particles showing same value for the measurement and 'anticoincidence' denotes those with opposite values. The defect in the local realistic theories is that they try to calculate this correlation essentially by averaging over the products of eigenvalues. Obviously the phase information is thrown away in this procedure and there is no way, conceptually, such an attempt would have reproduced quantum mechanical results. We calculate the experimenter's correlations starting from local amplitudes.

Now we state the expressions for the amplitudes and the amplitude correlation function. This is new physical input [5].

1. The local amplitudes for transmission associated with the first particle is $C_{1+}=$ $\frac{1}{\sqrt{2}} \exp (i \theta s)$ for measurements at analyzer1, and for the second particle it is $C_{2+}=\frac{1}{\sqrt{2}} \exp \left(i \theta^{\prime} s\right)$ at analyzer2. The amplitudes for the orthogonal outcome are $C_{1-}$ and $C_{2-}$ and these are rotated by $\pi / 2$ in the complex plane from the amplitudes for transmission. The square of the amplitudes give the corresponding probabilities $\left(C_{1+} C_{1+}^{*}\right.$ gives the probability for transmission, for example). $s$ is the spin of the particle ( 1 for photons and $\frac{1}{2}$ for the spin- $\frac{1}{2}$ singlet state- see below)

2. The amplitude correlation function is a normalized inner product of the amplitudes. This is of the form

$$
U(\mathbf{a}, \mathbf{b})=\operatorname{Real}\left(N C_{i} C_{j}^{*}\right)
$$

where $N$ is the normalization constant. The square of the amplitude correlation function gives the joint probabilities for events of the form $(++),(--)$, 
$(+-)$, and $(-+)$. All probabilities are guaranteed to be positive definite in our formalism since the amplitude correlation function is real.

The crucial difference from local realistic theories is that the correlation is calculated from quantities which preserve the relative phases.

Let us consider the maximally entangled singlet system described by Eq. 1, the most widely discussed example in the context of nonlocality. We prescribe the local amplitudes as $C_{1+}=\frac{1}{\sqrt{2}} \exp \left\{i s\left(\theta_{1}-\phi_{1}\right)\right\}$ for the first particle at the first polarizer and $C_{2+}=\frac{1}{\sqrt{2}} \exp \left\{i s\left(\theta_{2}-\phi_{2}\right)\right\}$ for the second particle at the second polarizer. There are corresponding amplitudes, $C_{1-}$ and $C_{2-}$ for the events denoted by -, and they differ only in the phase for the maximally entangled state.

The explicit dependence of the amplitude on the spin of the particle is motivated by the fact that we are dealing with systems with phases and the phase associated with the spin rotations (a geometric phase) is a necessary input in this description [7]. The correlation at source is encoded in $\phi_{0}$. The locality assumption is strictly enforced since the two amplitudes depend only on local variables and on an internal variable generated at the source and then individually carried by the particles without any subsequent interaction of any sort. The individual measurements at each end separately will now give the correct result for transmission for any angle of orientation. These probabilities are

$$
C_{1} C_{1}^{*}=C_{2} C_{2}^{*}=\frac{1}{2}
$$

Events of both types $(++)$ and $(--)$ contribute to a "coincidence". The correlation function for an outcome of either $(++)$ or $(--)$ of two maximally entangled particles is

$$
U\left(\theta_{1}, \theta_{2}, \phi_{o}\right)=2 \operatorname{Re}\left(C_{1} C_{2}^{*}\right)=\cos \left\{s\left(\theta_{1}-\theta_{2}\right)+s \phi_{o}\right\} .
$$

It is normalized such that its square will give the conditional joint probabilities of the type 'outcome + for the second particle, given that the outcome for the first particle is + , etc. All references to the individual values of the internal variable $\phi$ has dropped out.

We now derive the relation between this correlation function and the experimenter's correlation function $P(\mathbf{a}, \mathbf{b})=\frac{1}{N} \sum\left(A_{i} B_{i}\right)$. Since $U_{++}^{2}=U_{--}^{2}$ for the maximally entangled state, $U^{2}\left(\theta_{1}, \theta_{2}, \phi_{o}\right)$ is the probability for a coincidence detection ( ++ or --), and $\left(1-U^{2}\left(\theta_{1}, \theta_{2}, \phi_{o}\right)\right)$ is the probability for an anticoincidence (events of the type +- and -+ ). Since the average of the quantity (number of coincidences number of anticoincidences) $=$

$$
U^{2}\left(\theta_{1}, \theta_{2}, \phi_{o}\right)-\left(1-U^{2}\left(\theta_{1}, \theta_{2}, \phi_{o}\right)\right)=2 U^{2}\left(\theta_{1}, \theta_{2}, \phi_{o}\right)-1,
$$

the correspondence between $P(\mathbf{a}, \mathbf{b})$ and $U\left(\theta_{1}, \theta_{2}, \phi_{o}\right)$ is given by the expression,

$$
\begin{aligned}
P(\mathbf{a}, \mathbf{b}) & =2 U^{2}\left(\theta_{1}, \theta_{2}, \phi_{o}\right)-1 \\
& =2 \cos ^{2}\left\{s\left(\theta_{1}-\theta_{2}\right)+s \phi_{o}\right\}-1
\end{aligned}
$$


This completes the back-bone of our formalism and we are ready to discuss some specific examples.

\section{Spin- $\frac{1}{2}$ particles and Photons}

Consider the singlet state breaking up into two spin- $\frac{1}{2}$ particles propagating in opposite directions to spatially separated regions. Since orthogonality of the two particles in any basis implies a relative angle of $\pi$ for spinors, we set $\phi_{o}=\pi$. Then the correlation function and $P(\mathbf{a}, \mathbf{b})$ calculated from this function are

$$
\begin{aligned}
U\left(\theta_{1}, \theta_{2}, \phi_{o}\right) & =\cos \left\{s\left(\theta_{1}-\theta_{2}\right)+s \phi_{o}\right\} \\
& =\cos \left\{\frac{1}{2}\left(\theta_{1}-\theta_{2}\right)+\pi / 2\right\} \\
& =-\sin \frac{1}{2}\left(\theta_{1}-\theta_{2}\right) \\
P(\mathbf{a}, \mathbf{b})= & 2 \sin ^{2}\left(\frac{1}{2}\left(\theta_{1}-\theta_{2}\right)\right)-1 \\
& =-\cos \left(\theta_{1}-\theta_{2}\right)=-\mathbf{a} \cdot \mathbf{b}
\end{aligned}
$$

This is identical to the quantum mechanical predictions obtained from the singlet entangled state and Pauli spin operators. We have reproduced the correct correlation function using local amplitudes.

For the case of photons entangled in orthogonal polarization states we get, by setting $s=1$ and $\phi_{o}=\pi / 2$ to represent orthogonal polarization,

$$
\begin{gathered}
\begin{array}{c}
U\left(\theta_{1}, \theta_{2}, \phi_{o}\right)=\cos \left\{\left(\theta_{1}-\theta_{2}\right)+\pi / 2\right\} \\
=-\sin \left(\theta_{1}-\theta_{2}\right)
\end{array} \\
P(\mathbf{a}, \mathbf{b})=2 \sin ^{2}\left(\theta_{1}-\theta_{2}\right)-1=-\cos \left(2\left(\left(\theta_{1}-\theta_{2}\right)\right)\right.
\end{gathered}
$$

which is the correct quantum mechanical correlation.

The same analysis works for particles entangled in other sets of variables like momentum and coordinate, and energy and time. These cases of two particle entanglement can be mapped on to the spin- $\frac{1}{2}$ singlet problem with two-valued outcomes. Starting from the local amplitudes $\stackrel{C}{C}_{1}=\frac{1}{\sqrt{2}} \exp \left(i \alpha k\left(x_{1}-x_{o}\right) / 2\right)$, and $C_{2}=$ $\frac{1}{\sqrt{2}} \exp \left(i \alpha k\left(x_{2}-x_{o}\right) / 2\right)$ we can derive the probability for coincidence detection as

$$
P\left(x_{1}, x_{2}\right)=\cos ^{2}\left(\alpha k\left(x_{1}, x_{2}\right) / 2\right)=\frac{1}{2}\left(1+\cos k \alpha\left(x_{1}-x_{2}\right)\right)
$$


This is the two photon correlation pattern with $100 \%$ visibility, obtained without nonlocality. $x_{1}$ and $x_{2}$ are the coordinates of the two detectors separated by a spacelike interval. $k$ is the wave vector and $\alpha$ is a scaling factor for the angle subtended by the two slits at the detectors, source etc. The factor 2 dividing the angular variable comes from the mapping with the spin- $\frac{1}{2}$ problem.

\section{Three-particle GHZ correlations}

The three particle G-H-Z state [8] is defined as

$$
\left|\Psi_{G H Z}\right\rangle=\frac{1}{\sqrt{2}}(|1,1,1\rangle-|-1,-1,-1\rangle)
$$

where the eigenvalues in the kets are with respect to the $z$-axis basis.

The prediction from quantum mechanics for the measurement represented by the operator $\sigma_{x}^{1} \otimes \sigma_{x}^{2} \otimes \sigma_{x}^{3}$ is given by

$$
\sigma_{x}^{1} \otimes \sigma_{x}^{2} \otimes \sigma_{x}^{3}\left|\Psi_{G H Z}\right\rangle=-\left|\Psi_{G H Z}\right\rangle
$$

Equivalently the joint probabilities for various outcomes in the $x$ direction are

$$
\begin{aligned}
& P(+,+,+)=P(-,-,+)=P(+,-,-)=P(-,+,-)=0 \\
& P(-,-,-)=P(+,+,-)=P(+,-,+)=P(-,+,+)=1
\end{aligned}
$$

Local realistic theories predict that the product of the outcomes in the $x$ direction for the three particles should be +1 , i.e.e,

$$
P(+,+,+)=P(-,-,+)=P(+,-,-)=P(-,+,-)=1
$$

This contradicts Eqs. 15-17 and highlights the conflict between a local realistic theory and quantum mechanics.

The solution using local amplitudes is simple and physically revealing [9]. We define the local amplitudes for the outcomes + and - at the analyzer (with respect to the $x$ basis) for the first particle as $C_{1+}=\frac{1}{\sqrt{2}} \exp \left(i \theta_{1}\right)$, and $C_{1-}=\frac{1}{\sqrt{2}} \exp \left(i\left(\theta_{1}+\pi / 2\right)\right)$. The amplitude $C_{1-}$ contains the added angle $\pi / 2$ because this amplitude is orthogonal to $C_{1+}$. Similarly, we have $C_{2+}=\frac{1}{\sqrt{2}} \exp \left(i \theta_{2}\right)$, and $C_{2-}=\frac{1}{\sqrt{2}} \exp \left(i\left(\theta_{2}+\pi / 2\right)\right)$ for the second particle and $C_{3+}=\frac{1}{\sqrt{2}} \exp \left(i \theta_{3}\right)$, and $C_{3-}=\frac{1}{\sqrt{2}} \exp \left(i\left(\theta_{3}+\pi / 2\right)\right)$ for the third particle.

Correlation function is obtained from $N \operatorname{Real}\left(C_{1} C_{2}^{*} C_{3}^{*}\right)$, where $N$ is a normalization constant, and its square is the relevant joint probability. (There is no unique definition of the amplitude correlation function. The final results are independent of 
the particular definition we use). Since we want $N \operatorname{Re}\left(C_{1_{-}} C_{2-}^{*} C_{3_{-}}^{*}\right)= \pm 1$, we choose $C_{1-} C_{2-}^{*} C_{3-}^{*}$ to be pure real. This gives

$$
\begin{gathered}
\frac{N}{2 \sqrt{2}} \operatorname{Real}\left(\exp i\left(\theta_{1}-\theta_{2}-\theta_{3}-\pi / 2\right)\right)= \pm 1 \\
\theta_{1}-\theta_{2}-\theta_{3}-\pi / 2=0 \text { or } \pm \pi
\end{gathered}
$$

We can choose the relevant relative phases to satisfy this condition. Then we get

$$
P(-,-,-)=1
$$

Rest of the joint probabilities given in Eq. 6 automatically follow, since flipping sign once rotates the complex number $C_{1-} C_{2-}^{*} C_{3-}^{*}$ through $\pi / 2$. The square of $N$ Real $\left(C_{1} C_{2}^{*} C_{3}^{*}\right)$ is then 1 for an odd number of $(-)$ outcomes and 0 for even number of $(-)$ outcomes.

Similar construction also applies to four- particle maximally entangled state [10] and general multiparticle maximally entangled states.

\section{Concluding remarks}

We have also constructed local amplitudes for the Hardy experiment [11] in which quantum mechanics predicts three particular zero joint probabilities are one nonzero joint probability (the other possible joint probabilities in the problem can be nonzero and are not relevant for the demonstration of nonlocality). Local complex amplitudes that reproduce the four relevant joint probabilities can be constructed easily. It is impossible to achieve this if local realism at the level of eigenvalues are assumed.

We note that there is a simple way to physically understand the fact that the quantum correlations are typically larger than the corresponding classical correlations. The overlap (inner product) between a normalized random vector with $N$ elements and any basis vector is $1 / N$ for a classical vector, and $1 / \sqrt{N}$ for a quantum vector (amplitude). Therefore quantum correlations are typically stronger than classical correlations. In fact, it is this same physical fact that forms the basis of quantum search algorithms [12], where the initial overlap between a random vector and the desired basis vector is $\sqrt{N}$ times larger in the quantum case, making the search faster by $\sqrt{N}$.

The following table summarizes the locality and reality properties in various approaches to quantum correlations: 


\begin{tabular}{||l|l|l|l|l|l||}
\hline \hline $\begin{array}{l}\text { Theory/ } \\
\text { Formalism }\end{array}$ & $\begin{array}{l}\text { Basic } \\
\text { quantity }\end{array}$ & Locality & Reality & Determinism & Predictions \\
\hline \hline $\begin{array}{l}\text { Quantum } \\
\text { mechanics }\end{array}$ & $\begin{array}{l}\text { Multiparticle } \\
\text { wavefunction }\end{array}$ & NO & NO & NO & Correct \\
\hline $\begin{array}{l}\text { Local Real- } \\
\text { istic theories } \\
\text { with hidden } \\
\text { variables }\end{array}$ & Eigen values & YES & YES & YES & Incorrect \\
\hline $\begin{array}{l}\text { Present } \\
\text { formalism }\end{array}$ & Amplitudes & YES & $\begin{array}{l}\text { Yes (for } \\
\text { phase) }\end{array}$ & NO & Correct \\
\hline
\end{tabular}

Quantum entanglement swapping [13] is understood within this frame work by noting that Bell state measurements choose subensembles of particle pairs that show a particular joint outcome. Particles entangled independently with the pair of particles that are subjected to the Bell state measurement will show a joint outcome consistent with swapped entanglement due to the correlation encoded in the internal variable. But the Bell state measurement does not collapse the distant particle into a definite state. Yet all correlations are correctly reproduced. This has important implication to the interpretation of quantum teleportation. The present nonlocal interpretation of quantum teleportation is not correct.

In summary, the long standing puzzle of nonlocality in the EPR correlations is resolved. There is no nonlocal influence between correlated particles separated into space-like regions. The solution has new physical and philosophical implications regarding the nature of reality, measurement and state reduction in quantum systems.

\section{References}

[1] A. Einstein, B. Podolsky, and N. Rosen, Phys. Rev., 47, 777, (1935).

[2] J. S. Bell, Physics, 1, 195, (1965).

[3] A recent resource for exhaustive search is the recent book, The Einstein, Podolsky, and Rosen Paradox in Atomic, Nuclear and Particle Physics, A. Afriat and F. Selleri, (Plenum Press, New York and London), 1999.

[4] See for example, R. Penrose, Emperor's new mind, pp 370-371, (Vintage, UK, 1990).

[5] C. S. Unnikrishnan, There is no spooky action-at-a-distance in quantum correlations: Resolution of the EPR nonlocality puzzle, quant-ph/0001112, (2000).

[6] D. Bohm, Quantum Theory, (Prentice Hall, Englewood Cliffs, NJ (1951). 
[7] C. S. Unnikrishnan, to be published; The geometric phase associated with spin rotations can be obtained directly from a generalization of the usual dynamical phase $\int p_{i} d x^{i}$, to include the spin as one of the momenta $p_{i}$ and the angular coordinate as the corresponding $d x^{i}$.

[8] D. M. Greenberger, M. Horne, and A. Zeilinger in Bell's Theorem, Quantum Theory and Conceptions of the Universe, Ed. M. Kafatos, (Kluwer, Dordrecht,1989), pp 73-76 ; N. D. Mermin, Phys. Today, June 1990, p 9-11; D. M. Greenberger, M. Horne, A. Shimony, and A. Zeilinger, Am. J. Phys. 58, 1131 (1990).

[9] C. S. Unnikrishnan, Three-particle GHZ correlations without nonlocality, quantph/0004089, (2000).

[10] P. Hariharan, J. Samuel, and Supurna Sinha, J. Opt. B: Quantum Semiclass.Opt., 1, 199 (1999).

[11] L. Hardy, Phys. Rev. Lett., 71, 1665 (1993).

[12] L. K. Grover, Phys. Rev. Lett., 78, 325, (1997).

[13] C. H. Bennet et al, Phys. Rev. Lett., 70, 1895 (1993); M. Zukowski, A. Zeilinger, M. A. Horne, and A. Ekert, Phys. Rev. Lett., 71, 4287 (1993); J-W. Pan, D. Bouwmeester, H. Weinfurter and A. Zeilinger, Phys. Rev. Lett., 80, 3891 (1998). 